\title{
Cell Selection in 4G Cellular Networks
}

\author{
David Amzallag, Reuven Bar-Yehuda, Danny Raz, and Gabriel Scalosub
}

\begin{abstract}
Cell selection is the process of determining the cell(s) that provide service to each mobile station. Optimizing these processes is an important step toward maximizing the utilization of current and future cellular networks. We study the potential benefit of global cell selection versus the current local mobile SNR-based decision protocol. In particular, we study the new possibility available in OFDMA-based systems, such as IEEE $802.16 \mathrm{~m}$ and LTE-Advanced, of satisfying the minimal demand of a mobile station simultaneously by more than one base station. We formalize the problem as an optimization problem, and show that in the general case this problem is not only NP-hard but also cannot be approximated within any reasonable factor. In contrast, under the very practical assumption that the maximum required bandwidth of a single mobile station is at most an $r$-fraction of the capacity of a base station, we present two different algorithms for cell selection. The first algorithm produces a $(1-r)$-approximate solution, where a mobile station can be covered simultaneously by more than one base station. The second algorithm produces a $\frac{1-r}{2-r}$-approximate solution, while every mobile station is covered by at most one base station. We complete our study by an extensive simulation study demonstrating the benefits of using our algorithms in high-loaded capacity-constrained future 4G networks, compared to currently used methods. Specifically, our algorithms obtain up to 20 percent better usage of the network's capacity, in comparison with the current cell selection algorithms.
\end{abstract}

Index Terms-Cellular networks, 4G, WiMAX, LTE-advanced, approximation algorithms, cell selection, association, resource allocation

\section{INTRODUCTION}

THE ability to provide services in a cost-effective manner 1 is one of the most important building blocks of competitive modern cellular systems. Usually, an operator would like to have a maximal utilization of the installed equipment, that is, to maximize the number of satisfied customers at any given point in time. This paper addresses one of the basic problems in this domain, the cell selection mechanism. This mechanism determines the base station (or base stations) that provides the service to a mobile station-a process that is performed when a mobile station joins the network (called cell selection), or when a mobile station is on the move in idle mode (called cell reselection, or cell change, in HSPA).

In most current cellular systems the cell selection process is done by a local procedure initialized by a mobile device according to the best detected SNR. In this process, the mobile device measures the SNR to several base stations that are within radio range, maintains a "priority queue" of those that are best detected (called an active set), and sends an official service subscription request to base stations by their order in that queue. The mobile station is connected to the first base station that positively confirmed its request. Reasons for rejecting service requests may be handovers or drop-calls areas, where the capacity of the base station is

- D. Amzallag is with Amdocs Ltd., 2 HaElla Street, Ramat Efal 52960, Israel.E-mail: david.amzallag@amdocs.com.

- R. Bar-Yehuda and D. Raz are with the Computer Science Department, Technion, Haifa 32000, Israel. E-mail: \{reuven, danny\}@cs.technion.ac.il.

- G. Scalosub is with Communication Systems Engineering, Ben-Gurion University of the Negev, P.O.B. 653, Beer-Sheva 84105, Israel.

E-mail: sgabriel@bgu.ac.il.

Manuscript received 27 Feb. 2011; revised 9 Nov. 2011; accepted 16 Mar. 2012; published online 28 Mar. 2012.

For information on obtaining reprints of this article, please send e-mail to: tmc@computer.org, and reference IEEECS Log Number TMC-2011-02-0102. Digital Object Identifier no. 10.1109/TMC.2012.83. nearly exhausted. Such approaches usually result in significantly suboptimal associations of mobile users to base stations. Optimizing cell selection is crucial in future 4G networks due to both the restricted capacity available at the base stations (e.g., in the case of femtocells), combined with the increased demand by the mobile users. To the best of our knowledge, very little work has focused on optimizing these procedures taking into account the scarcity of spectrum expected in future $4 \mathrm{G}$ cellular networks.

Consider, for example, the settings depicted in Fig. 1. Assume that the best SNR for Mobile Station 1 (MS1) is detected from microcell A, and thus MS1 is being served by this cell. When Mobile Station 2 (MS2) arrives, its best SNR is also from microcell $\mathrm{A}$, who is the only cell able to cover MS2. However, after serving MS1, microcell A does not have enough capacity to satisfy the demand of MS2 who is a heavy data client. However, if MS1 could be served by picocell B then both MS1 and MS2 could be served. Note that MS1 and MS2 could represent a cluster of clients. The example shows that the best-detected-SNR algorithm can be a factor of $\max \{\tilde{d}\} / \min \{\tilde{d}\}$ from an optimal cell assignment, where $\tilde{d}$ is the demand of any mobile station in the coverage area. Theoretically speaking, this ratio can be arbitrarily large.

This simple example illustrates the need for a global, rather then a local, cell selection solution that tries to maximize the global utilization of the network, and not just the SNR of a single user. In voice only networks, where base station capacities are considered to be high, sessions have limited duration, and user demands are uniform, this may not be a big barrier. That is, the current base station selection process results, in most cases, in a reasonable utilization of the network. However, in the forthcoming $4 \mathrm{G}$ cellular networks this may not be the case. 


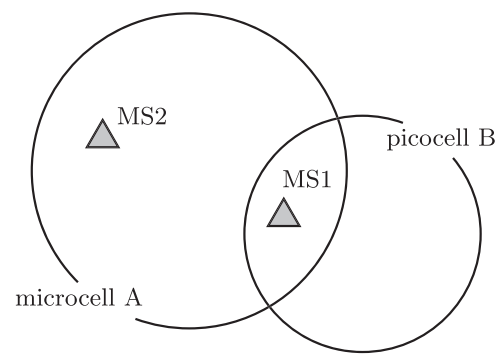

Fig. 1. Bad behavior of the best detected SNR algorithm in high-loaded capacitated network.

Although the detailed structure of $4 \mathrm{G}$ systems is as of yet not well defined, there is a clear consensus regarding some of the important aspects of the technologies to be implemented in these systems. ${ }^{1}$ Fourth generation systems are planned to provide even higher transmission rates and larger capacity than current 3G (IMT-2000 based) systems, both in terms of the number of users as well as in terms of traffic volume. Most likely, 4G systems will be designed to offer bit rates of $100 \mathrm{Mbit} / \mathrm{s}$ (peak rate in mobile environment) to $1 \mathrm{Gbit} / \mathrm{s}$ (fixed indoors) with a $5 \mathrm{MHz}$ frequency bandwidth. The systems' capacities are expected to be at least 10 times larger than current 3G systems. In addition, these objectives should be met together with a drastic reduction in the cost (1/10 to $1 / 100$ per bit) [20]. Such high frequencies yield a very strong signal degradation and suffer from significant diffraction resulting from small obstacles, hence forcing the reduction of cell size (in order to decrease the amount of degradation and to increase the degree of coverage), resulting in a significantly larger number of cells compared to previous generations. In addition, with the further deployment of technologies such as femtocells in providing broadband access, it is expected that users will remain connected for long periods of time. When considering the user demand to cell capacity ratio, for voice traffic in current cellular technologies, a single cell may support up to $\sim 100$ concurrent voice connections [17]. As demands are expected to increase on the order of two- to three fold, whereas capacities are expected to increase by a mere one- to two fold [23], the resulting user demand to cell capacity ratio is expected to increase by at least one order of magnitude. These facts render the optimization of cell selection and association extremely significant in optimizing spectrum and capacity utilization.

The increased number of base stations, and the variable bandwidth demand of mobile clients, will force operators to optimize the way the capacity of a base station is utilized. Unlike in previous generations, the ability of a base station to successfully satisfy the service demand of all its mobile clients would be highly limited and will mostly depend on its infrastructure restrictions, as well as on the service distribution among its mobile clients.

Another interesting aspect is the support for different QoS classes for the mobile stations, (e.g., gold, silver, or bronze). In such a case, the operator would like to have as many satisfied "gold" customers as possible, even if this means several unsatisfied "bronze" customers.

1. See the International Telecommunication Union (ITU) website at http://www.itu.int/home/index.html.
In this paper, we study the potential benefit of a new global cell selection mechanism, which should be contrasted with the current local mobile SNR-based decision protocol. In particular, we rigourously study the problem of maximizing the number of mobile stations that can be serviced by a given set of base stations in such a way that each of the serviced mobile stations has its minimal demand fully satisfied. More formally, we consider a collection of users, each having some demand (e.g., in terms of rate) and some profit, alongside a set of base stations, each with some capacity (e.g., in terms of available bandwidth). Each base station has some coverage area, modeled by a set of clients it can potentially service. We focus our attention on potentially overloaded settings, where it might not be possible for the base stations to meet the demands of all the users, due to capacity constraints. Our goal is to find a fractional assignment of users to base stations, such that the overall profit obtained by users that are fully covered, is maximized. We differentiate between two coverage paradigms: The first is cover-by-one where a mobile station can receive service from at most one base station. This paradigm is the common case considered in previous work (e.g., the generalized assignment problems, which is discussed in detail in Section 3). In this coverage approach the produced assignment is actually integral. The second paradigm, which is the main focus of our work, is cover-by-many, where we allow a mobile station to be simultaneously satisfied by more than one base station. This means that when a mobile stations has a relatively high demand (e.g., video-on-demand) in a sparse area (e.g., sea shore), several base stations from its active set can participate in its demand satisfaction. This option is not available in third-generation networks (and not even in HSPA networks) since these networks have universal frequency reuse and the quality of a service a mobile station receives will be severely damaged by the derived cochannel interference. However, OFDMA-based technology systems and their derivatives are considered to be among the prime candidates for future cellular communication networks. The ability to satisfy the demand of a mobile station by more than one member of its active set is possible in these systems, as defined by the IEEE 802.16m standard, using MIMO technology [2], and also as part of LTE-Advanced (e.g., in the form of cooperative multipoint transmission) [1], [16]. An important question in this context is whether cover-by-many is indeed more powerful than cover-by-one, in the sense that it improves the ability of the network to satisfy more clients. Simple examples can show that improvement can indeed be made, by considering, e.g., combining the leftover capacities of base stations in order to support additional users. However, it is not clear how much more powerful is the cover-by-many paradigm, when compared with the cover-by-one approach.

Our work focuses on the offline version of the problem, where the entire input is given in advance. We believe that from a theoretical point of view, a better understanding of the offline problem is extremely helpful if one is to design good algorithms for distributed or online settings, which are the natural settings in which our problem is cast. For example, we note that our offline hardness results guide us to focus on restricted settings. Furthermore, additional work that followed the preliminary version of our work has 
built on our offline results to design distributed algorithms for related models, essentially focusing on the cover-by-one paradigm [25].

Approximation algorithms and heuristics play a major role in our paper. A $\gamma$-approximation algorithm is a polynomial-time algorithm that always finds a feasible solution for which the value of the objective function is within a proved factor of $\gamma$ of the optimal solution. Heuristics will be described in comparison with the worst case behavior of approximation algorithms, in order to design a good practical solution to the problems in question.

\subsection{Our Contribution}

In this paper, we present a new approach for cell selection that is derived from the anticipated $4 \mathrm{G}$ technologies. To the best of our knowledge, despite recent extensive research done on future cellular networks planning and coverage optimization (e.g., [4], [22]), there is no explicit study in the literature discussing the new IEEE $802.16 \mathrm{~m}$ and LTEAdvanced possibility of simultaneous servicing of mobile clients by more than one base station.

We model, in Section 2, the cell selection problem as an optimization problem called all-or-nothing demand maximization (AoNDM). We show that the general version of AoNDM cannot be approximated within a factor better than $|J|^{1-\epsilon}$, unless NP $=\mathrm{ZPP}$, for any $\epsilon>0$, where $J$ is the set of mobile stations. Motivated by this result, we address a special case of the problem. Following practical scenarios, we define a restrictive version of AoNDM, the $r$-AoNDM problem, for some $r<1$, where the network satisfies the condition that the demand of every mobile station is at most an $r$ fraction of the capacity of any base station that can potentially cover the mobile station. We show that even this special case of the problem is NP-hard. These results appear in Section 4.

We further present, in Section 4, two different algorithms for this problem. The first is a $\frac{1-r}{2-r}$-approximation algorithm, which uses the cover-by-one paradigm, i.e., every mobile station is covered by at most one base station. Note that this approximation guarantee is with regard to the optimal cover-by-many assignment. The second algorithm uses the cover-by-many paradigm, where a mobile station can be covered simultaneously by more than one base station. It is a careful refinement of the first algorithm, and we prove it guarantees at least a $1-r$ fraction of the value of an optimal solution, at a price of increased running time. We note that better approximation guarantees are known for the coverby-one assignment problem, however most of these algorithms were based on solving a linear programming relaxation of the problem and rounding the resulting solution in order to obtain an integral solution. Our algorithms, based on the local-ratio method, are combinatorial and provide further insight into the structure of the underlying problem. Furthermore, our results for the coverby-many assignment problem provide the currently best known guarantees for this problem.

In order to evaluate the practical differences between global and local mechanisms for cell selection in future networks we conducted an extensive simulation study (Section 5). We compare between global mechanisms that are based on our approximation algorithms and the current
best-SNR greedy cell selection protocol. We study the relative performance of these three algorithms under different conditions. In particular, we show that in a highload capacity-constrained 4G-like network, where clients' demands may be large with respect to cell capacity, global cell selection can achieve up to 20 percent better coverage than the current best SNR greedy cell selection method.

\section{Model and Definitions}

Consider a bipartite graph $G=(I, J, E)$ where $I=\{1,2$, $\ldots, m\}$ is the set of base stations and $J=\{1,2, \ldots, n\}$ is the set of mobile stations (or clients). Every client $j \in J$ has a nonnegative demand $d(j)$, and a nonnegative profit $p(j)$, and every base station $i \in I$ has a nonnegative capacity $c(i)$. In addition, for every base station $i \in I$, the coverage area of $i$ is modeled by a subset $S_{i} \subseteq J$ of clients which can be serviced by $i$. The set of base stations $N(j) \subseteq I$ connected by edges to a client $j \in J$, represents the active set of this client. We further extend the above definitions to sets of nodes, such that for every $A \subseteq J, d(A)=\sum_{j \in A} d(j)$, and $p(A)=$ $\sum_{j \in A} p(j)$, and for every $B \subseteq I, c(B)=\sum_{i \in B} c(i)$. Furthermore, given any $A \subseteq J$, we let $N(A)=\bigcup_{j \in A} N(j)$. Given a subset of clients $S \subseteq J$, a cover plan for $S$ is a weight function $x: E \rightarrow \mathbb{R}^{+}$, such that for every $j \in S, \sum_{i:(i, j) \in E} x(i, j) \geq d(j)$, and for every $i \in I, \sum_{j:(i, j) \in E} x(i, j) \leq c(i)$. Notice that such a restriction of $\sum_{i:(i, j) \in E} x(i, j) \geq d(j)$, for every $j \in S$, is also known as all-or-nothing-type of coverage. This means that clients that are partially satisfied are not considered to be covered (such a model appears, for example, in OFDMAbased networks where mobile stations have their slot requirements over a frame and these are not useful if not fulfilled).

The all-or-nothing demand maximization problem is to find a subset of clients $S \subseteq J$, and a cover plan $x$ for $S$, such that $p(S)$ is maximized.

For $i \in I$, we use $x(i)=\sum_{j:(i, j) \in E} x(i, j)$, and for $j \in J$, we use $x(j)=\sum_{i:(i, j) \in E} x(i, j)$. As before, we extend these notations to sets of nodes, such that for every $A \subseteq I$, $x(A)=\sum_{i \in A} x(i)$, and for every $B \subseteq J, x(B)=\sum_{j \in B} x(j)$. We further extend this notation to subgraphs of $G$, such that given any $A \subseteq I$ and $B \subseteq J, x(A, B)=\sum_{(i, j) \in E \cap(A \times B)} x(i, j)$.

In addition, for every $v \in I \cup J$ we denote by $E(v)$ the set of edges with endpoint $v$, and for every $W \subseteq I \cup J$, let $E(W)=\bigcup_{v \in W} E(v)$. We further denote for every $A \subseteq I$ and $B \subseteq J, E(A, B)=\{(i, j) \in E \cap(A \times B)\}$.

Given any constant $r<1$, we say an instance is $r$ restricted if for every $(i, j) \in E, d(j) \leq r \cdot c(i)$. We further define the problem of $r$-AoNDM as the AoNDM problem limited to $r$-restricted instances.

\section{Related Work}

Cell selection has received much attention in recent years (e.g., [14], [19], [26], [27]) where research focused mainly on multiple-access techniques, as well as on power control schemes and handoff protocols [14], [26], [27].

In [14], a cell selection algorithm is presented where the goal is to determine the power allocations to the various 
users, as well as a cover-by-one allocation, so as to satisfy per-user SINR constraints. An HSPA-based handoff/cellsite selection technique is presented in [26], [27], where the objective is to maximize the number of connected mobile stations (very similar to our objective), and reaching the optimality of this objective is done via a new scheduling algorithm for this cellular system. All the above results did not take into account variable base station capacities nor mobile station bandwidth demands. In the case of [26], [27], this enables the authors to reduce their corresponding optimization problem to a polynomial-time solvable matching problem. As shown in our paper, when base station capacities and/or mobile stations' demands are incorporated, this approach is no longer feasible.

An integrated model for optimal cell-site selection and frequency allocation is shown in [19], where the goal is to maximize the number of connected mobile stations, while maintaining quasi independence of the radio based technology. The optimization problem in this model is shown to be NP-hard.

The case where we restrict the clients in AoNDM to be satisfied by a single base station belongs to the family of generalized assignment problems. Among this class of problems the most related problem to AoNDM is the separable assignment problem (SAP) [13].

In this problem, we are given a set $U$ of $m$ bins, a set $H$ of $n$ items, and a profit, $f_{i j}$, for assigning item $j$ to bin $i$. The assignment constraints are such that every $i \in U$ has a family $\mathcal{I}_{i}$ of feasible subsets that can be packed in bin $i$, such that $\mathcal{I}_{i}$ is closed under taking subsets, i.e., if $A \in \mathcal{I}_{i}$, then so is every subset of $A$. The goal is to find an assignment of items to bins with the maximum aggregate profit.

The suggested SAP solution presented in [13], depends on an algorithm which solves the single-bin subproblem in SAP. Given a $\beta$-approximation algorithm for finding the highest profit packing of a single bin, they present a polynomial-time LP-rounding based $\left(\left(1-\frac{1}{e}\right) \beta\right)$-approximation algorithm and a polynomial-time local search $\left(\frac{\beta}{\beta+1}-\epsilon\right)$ - approximation algorithm, for any $\epsilon>0$. Specifically, for all special cases of SAP that admit an approximation scheme for the single-bin problem, there exists an LP-based algorithm with a $\left(1-\frac{1}{e}-\epsilon\right)$-approximation guarantee, and a local search algorithm with a $\left(\frac{1}{2}-\epsilon\right)$-approximation guarantee.

This problem is a generalization of several well-known problems. Among these problems are the maximum generalized assignment problem (GAP), and the multiple knapsack problem (MKP). In GAP, we are given a set of bins with capacity constraints and a set of items that have a possibly different size and profit for each bin. We wish to pack a maximum-profit subset of items into the bins. MKP is the special case of GAP where the size and the profit of each item are the same for all the bins.

Shmoys and Tardos [28] give an LP-rounding based 2-approximation algorithm for the minimization version of GAP. However, Chekuri and Khanna [8] observed that a 1/2-approximation for standard GAP is implicit in [28]. In addition, Chekuri and Khanna [8] develop a PTAS for MKP and also classify the APX-hardness of GAP. Cast in our terminology, the algorithm of Shmoys and Tardos [28] for the GAP problem solves our problem using the cover-by- one paradigm. Their algorithm, which is based on solving a linear programming (LP) relaxation of the problem, and rounding the solution to obtain an integral solution, guarantees a 2-approximate solution with respect to the optimal fractional solution to the LP, which also serves as an upper bound on the optimal solution to our problem under the cover-by-many paradigm. This result holds for any $r \leq 1$. Furthermore, for $r$-restricted instances with $r<1 / 2$, the observation made by Chekuri and Khanna [8] implies that the cover-by-one algorithm proposed by Shmoys and Tardos yields a $(1-2 r)$-approximate solution. Our scheme provides a twofold improvement upon these results; First, our algorithms are combinatorial, and do not resort to solving linear programming relaxations of the problem. Second, our ultimate result guarantees a $(1-r)$-approximate solution for any $r \leq 1$, which improves upon the best known results for any $r \leq 1 / 2$.

Fractional packings, where items are allowed to be partitioned among more than one bin, are studied in [21] and in [18]. The assumption in the models studied in these papers is that splitting an item is associated with overhead, thus the objective is to pack the items (possibly splitting some of the items) with maximal profit and minimum overhead. Our problem is different since in our case, while splitting is for free, not every item can be packed in every bin.

Combinatorial algorithms, and more specifically localratio and primal dual algorithms, which only allow an item to be packed in a single bin, have been considered for some of the above mentioned related problems. A greedy algorithm that iteratively assigns users to base stations using the best approximation scheme for the Knapsack problem, was proved to produce a $2+\epsilon$-approximation [11]. For the particular case considered in this paper, where a user cannot be covered by several base stations, a simple 2-approximation based on finding a maximum $s$ - $t$ flow was suggested in [24].

AoNDM is closely related to the problem of planning $4 \mathrm{G}$ cellular networks under budget limitation as described in [3], [5]. In this problem, in addition to the input of AoNDM, we are given a set $I$ of possible configuration of base stations, as well as an opening cost $w(i)$ for every $i \in I$. When a client belongs to the coverage area of more than one base station, interference between the servicing stations may occur. These interferences are modeled by a penaltybased mechanism and may reduce the contribution of a base station to a client. The budgeted cell planning problem asks for a subset of base stations $I^{\prime} \subseteq I$ whose cost does not exceed a given budget $B$, and the total number of fully satisfied clients is maximized. Notice that in these settings, by taking the set $I$ of base stations with zero opening costs, without interferences, we get a special case of AoNDM where all clients have the same profit. It was shown [3] that this problem cannot be approximated, unless $\mathrm{P}=\mathrm{NP}$, and that a $\frac{e-1}{3 e-1}$-approximation algorithm exists for a special case of the problem where every set of $k$ open base stations can fully satisfy at least $k$ clients, for every integral value of $k$.

Another closely related problem is the all-or-nothing multicommodity flow problem discussed in [9] and [10]. In this problem, we are given a capacitated undirected graph $G=$ $(V, E, u)$ (where $u$ is the edge-capacity function) and set of $k$ pairs $\left(s_{1}, t_{1}\right), \ldots,\left(s_{k}, t_{k}\right)$. Each pair has a unit demand. The objective is to find a largest subset $S$ of $\{1, \ldots, k\}$ such that 
one can simultaneously route for every $i \in S$ one unit of flow between $s_{i}$ and $t_{i}$. It is straightforward to verify that the unit profit version of AoNDM is a special case of this problem. It was shown that the all-or-nothing multicommodity flow problem can be approximated within an $O\left(\log ^{2} k\right)$ factor of the optimum [10]. On the other hand, for any $\epsilon>0$, the problem cannot be approximated to within a factor of $O\left(\log ^{\frac{1}{3}-\epsilon}|E|\right)$ of the optimum, unless NP $\subseteq$ ZPTIME $\left(|V|^{\text {poly } \log |V|}\right)$ [6]. However, no special attention is given to specific network topologies (e.g., bipartite graphs, as in our case), and other special instances.

Following the preliminary version of our work, PattShamir et al. [25] have considered our problem in the distributed setting, focusing merely on the cover-by-one paradigm. They build upon our results and show, for any $\epsilon \in(0,1)$, a distributed $\frac{1-r}{2-r}(1-\epsilon)$-approximation (with high probability) algorithm which runs in $O\left(\epsilon^{-2} \log ^{3} n\right)$ communication rounds. This result uses a distributed emulation of our proposed cover-by-one algorithm, which iteratively finds maximal matchings to improve upon the solution.

\section{Approximating the $r$-AondM Problem}

\subsection{Lower Bounds}

The important goal of efficiently solving the AoNDM problem is beyond our reach since this problem is NPhard, as we mentioned before. Moreover, as the following theorem shows, even obtaining a reasonable approximation algorithm for the problem is improbable under standard complexity assumptions.

Theorem 4.1. For any $\epsilon>0$, AoNDM cannot be approximated to within a factor better than $|J|^{1-\epsilon}$, unless $N P=Z P P$.

Proof. We present a reduction from the Maximum-Size Independent Set (MIS) problem to AoNDM. Let $G=$ $(V, E)$ be any input to MIS. Consider the bipartite graph $\tilde{G}=(I, J, \tilde{E})$, where $I=E, J=V$, and $\tilde{E}=\{(e, v) \in$ $E \times V \mid \mathrm{v}$ is an endpoint of e\}. For every $v \in V$, let $\delta(v)$ denote the degree of $v$ in $G$, and let $M=\max _{v} \delta(v)$. For every $j \in J$ we set $d(j)=\delta(j)$ and set $p(j)=1$. Finally, we define for every $i \in I, c(i)=1$.

Since all clients have unit profit, our goal is to maximize the number of clients which can be covered. Let $S$ be any subset of $J$, and let $x$ be any cover plan for $S$. For any $j \in S$, the overall capacity of the base stations connected to $j$ is

$$
\sum_{i:(i, j) \in \tilde{E}} c(i)=\delta(j)=d(j) .
$$

It follows, that any client covered in $S$ uses all the capacity of the base stations in its range. Hence, a base station may contribute to the covering of at most one client, and in particular, any $e \in E$ can contribute to covering at most one of its endpoints. It follows that for any $S \subseteq V, S$ has a cover plan if and only if it is an independent set in $G$. Since for any $\epsilon>0$, MIS cannot be approximated to within a factor better than $|V|^{1-\epsilon}$, unless $\mathrm{NP}=\mathrm{ZPP}$ [15], the same holds for AoNDM.

Note that in this reduction the client's demand is $\delta(j)$ which is greater than the capacity of each of the base stations $(=1)$. In realistic cellular networks this is not the case. Motivated by this fact, and the above theorem, we focus on a special case of the problem. Namely, for any $r<1$ we consider the $r$-AoNDM problem. The following theorem shows that even in such restrictive settings, the problem is still intractable.

Theorem 4.2. For any fixed $r<1$, the $r$-AoNDM problem is NP-hard, even if there is only one base station.

Proof. We show a reduction from the Knapsack problem, which is known to be NP-hard. Let $K$ be any instance to the Knapsack problem, which comprises of a set of elements $A$, and a knapsack of size $B$, such that every element $a \in A$ has a size $s(a)$, and a value $v(a)$. Let $S=\sum_{a \in A} s(a)$, and $P=\sum_{a \in A} v(a)$. Given any $r \in(0,1)$, we let $M \in \mathbb{N}$ such that $\frac{1}{M} \leq r<\frac{1}{M-1}$.

We construct a bipartite graph $G=(I, J, E)$, such that $I$ consists of a single node $i$, and $J=J_{A} \cup J_{B}$ where $J_{A}=\left\{j_{a} \mid a \in A\right\}$, and $J_{B}=\left\{b_{1}, \ldots, b_{M}\right\}$. We let $E=I \times$ $J$. For every $j_{a} \in J_{A}$ we let $d\left(j_{a}\right)=s(a)$, and $p\left(j_{a}\right)=v(a)$. For every $b_{\ell} \in J_{B}$ we let $d\left(b_{\ell}\right)=2 S$, and $p\left(b_{\ell}\right)=2 P$. We set $c(i)=2 M S+B$.

First note that the above instance to AoNDM is $r$-restricted. To see this note that for every $j \in J_{A}$, $d(j) \leq S<2 S+\frac{B}{M}$, and for every $j \in J_{B}, d(j)=2 S<$ $2 S+\frac{B}{M}$. Since $2 S+\frac{B}{M}=\frac{c(i)}{M} \leq r \cdot c(i)$ we have for every $(i, j) \in E, d(j) \leq r \cdot c(i)$.

In addition, note that for every optimal solution $X$ to the above $r$-AoNDM instance, $J_{B} \subseteq X$. This follows from the fact that $J_{B}$ is a feasible solution, and for every $j \in J_{B}$, the profit obtained by covering $j$ is strictly greater than the profit obtained from covering all of $J_{A}$. It therefore follows that the cover plan for $X$ uses exactly $2 M S$ units of $i$ 's capacity, leaving a capacity of $B$ to cover clients in $J_{A}$. Hence, the subset $X \cap J_{A}$ induces an optimal solution to the original knapsack problem. This completes the proof that $r$-AoNDM is NP-hard for any $r<1$, even if there is only one base station.

\subsection{Algorithmic Framework}

In the following sections, we present two approximation algorithms for the $r$-AoNDM problem. The algorithms are local-ratio algorithms that are based on a decomposition of the profit obtainable from every client into two nonnegative terms; One part is proportional to the demand of the client, while the other part is the remaining profit. We define a family of feasible solutions, which we dub "maximal" (see below for the formal definition), and prove that any such solution is an approximate solution when considering a profit function which is proportional to the demand. The algorithms we present generate such maximal solutions recursively. We then apply an inductive argument which proves that the solution generated by the algorithm is also an approximate solution w.r.t. the original profit function.

We first present an approximation algorithm that guarantees a solution whose value is within a factor of $\frac{1-r}{2-r}$ from the value of an optimal solution. This algorithm follows the cover-by-one paradigm, and thus every mobile station is covered by at most one base station. Our second algorithm is obtained by a careful refinement of this algorithm, and an appropriate change to the notion of maximality. This algorithm uses the cover-by-many paradigm, and is guaranteed to produce a solution whose value 
is within a factor of $(1-r)$ from the value of an optimal solution, while the complexity increases by a polynomial factor. Next, we specify several definitions needed for the analysis of the proposed algorithms.

Given any instance of $r$-AoNDM over a graph $G=$ $(I, J, E)$, and any two subsets $A \subseteq I$ and $B \subseteq J$, we define the $A$ - $B$ flow-graph of $G, G_{f}(A, B)=(V, F)$, such that $V=$ $\{s\} \cup A \cup B \cup\{t\}$ for new vertices $s, t \notin I \cup J$, and $F=$ $(\{s\} \times A) \cup E(A, B) \cup(B \times\{t\})$. We define a capacity function $\gamma: F \rightarrow \mathbb{R}^{+}$as follows:

$$
\gamma(u, v)=\left\{\begin{array}{cl}
c(v) & \text { if } u=s, v \in A \\
\infty & \text { if } u \in A, v \in B \\
d(u) & \text { if } u \in B, v=t
\end{array}\right.
$$

For brevity of notation, we let $G_{f}=G_{f}(I, J)$. Given any two subsets $C, D \subseteq V$, we let $\gamma(C, D)=\sum_{u, v \in F \cap(C \times D)} \gamma(u, v)$.

A cover plan $x$ for $S \subseteq J$ is said to be a cover-by-one plan if for every $j \in S$, there is exactly one $i \in I$ such that $x(i, j)>0$. Given a cover-by-one plan $x$ for $S \subseteq J$, a coverby-one plan $x^{\prime}$ for $T \subseteq J$ is said to be a $T$-extension of $x$, if for any $j \in S$ and every $i \in I, x^{\prime}(i, j)=x(i, j)$. Note that in such a case one is guaranteed to have $S \subseteq T$. Given a cover plan $x$ for $S \subseteq J$, a cover plan $x^{\prime}$ for $T \subseteq J$ is said to be a $T$ rearrangement of $x$, if $S \subseteq T$.

Given any cover-by-one plan $x$ for $S \subseteq J$, we say that $x$ is cover-by-one-maximal (CBO-maximal) if for any $j \in J \backslash S$, no $S \cup\{j\}$-extension of $x$ exists. We further say $S \subseteq J$ is CBO-maximal when it has a CBO-maximal cover plan which is clear from the context. For any $A \subseteq I$ and $B \subseteq J$, and any flow $y$ in $G_{f}(A, B)$, we can denote the value of the flow by $y(s)$. Given any cover plan $x$ for $S \subseteq J$, we say that $x$ is rearrangement-maximal if for any $j \in J \backslash S$, no $S \cup\{j\}$-rearrangement of $x$ exists. Given any set $S \subseteq J$, let $\bar{S}=J \backslash S$ and $Y_{S}=I \backslash N(\bar{S})$. We say a cover plan $x$ for $S \subseteq J$ is cover-by-many-maximal (CBM-maximal) if $x$ is rearrangement-maximal, and $x\left(Y_{S}, S\right)$ is a maximum flow in the flow graph $G_{f}\left(Y_{S}, S\right)$. As before, we further say $S \subseteq$ $J$ is CBM-maximal when it has a CBM-maximal cover plan which is clear from the context.

The following lemma, appearing in [7], serves as a basic tool with which we analyze the approximation guarantee of the algorithms proposed in this section.

Lemma 4.3 (Local Ratio). Let $\mathcal{I}$ be an instance to $r$-AoNDM, over a graph $G=(I, J, E)$, with profit function $p$. Then, if $p=p_{1}+p_{2}$, and $x$ is a cover plan for some set $S \subseteq J$ which is c-approximate w.r.t. $p_{1}$, and also c-approximate w.r.t. $p_{2}$, then $x$ is c-approximate w.r.t. $p$.

\subsection{A Cover-by-One $\frac{1-r}{2-r}$-Approximation Algorithm}

We start with Algorithm CBO-MC. We note that there are at hand cover-by-one algorithms that ensure an approximation ratio better than our proposed algorithm [8], [28]. However, these algorithms are based on solving linear programming relaxations of the problem, and rounding the resulting solution in order to obtain an integral solution. Our algorithm, on the other hand, is strictly combinatorial. Furthermore, our cover-by-one algorithm forms the basis for the algorithm we describe in Section 4.4, which uses the cover-by-many paradigm. In this sense, understanding the simpler settings set forth in this section also serves to clarify many of the tools and approaches used when describing and analyzing the more complex settings of the cover-bymany algorithm discussed in the sequel.

We now turn to describe our cover-by-one algorithm, Algorithm CBO-MC. Roughly speaking, under CBO-MC, given a specific ordering of the clients, and given an existing cover plan $x$, a client is added greedily by finding a CBOextension of $x$, if such an extension exists. Otherwise, the client is discarded. See Algorithm 1 for the pseudocode of the algorithm.

Algorithm 1. CBO-MC ( $G=(I, J, E)$, demands $d$, profits $p$, capacities $c$ ).

1: if $J=\emptyset$ then

2: $\quad$ return $x \equiv 0$

3: end if

4: if there exists a $j \in J$ such that $p(j)=0$ then

5: $\quad x \leftarrow$ CBO-MC $\left(G^{\prime}=(I, J \backslash\{j\}, E \backslash E(j)), d, p, c\right)$

6: $\quad$ return $x$

7: else

8: $\quad$ for every $j \in J$, set $\epsilon_{j}=\frac{p(j)}{d(j)}$

9: $\quad$ set $\epsilon=\min _{j} \epsilon_{j}$

10: $\quad$ for every $j \in J$, set $p_{1}(j)=\epsilon \cdot d(j)$

11: $\quad$ set $p_{2}=p-p_{1}$

12: $\quad x \leftarrow$ CBO-MC $\left(G, d, p_{2}, c\right)$

13: $\quad$ for every $j$ such that $p_{2}(j)=0$ do

14:

$15:$

16:

17:

18:

19:

20:

21: end if

if $\exists i \in N(j)$ such that $c(i)-x(i) \geq d(j)$ then

$$
\text { set } x(i, j)=d(j)
$$

else

discard $j$

end if

end for

return $x$

Lemma 4.4. Consider any instance of the $r$-AoNDM problem such that for every client $j, p(j)=\epsilon \cdot d(j)$, for some constant $\epsilon$. Any cover-by-one plan $x$ for $S \subseteq J$ which is CBO-maximal is a $\frac{1-r}{2-r}$-approximate solution w.r.t. profit function $p$. Furthermore, this approximation is with regards to the optimal cover-by-many solution.

Proof. Let $\bar{S}=J \backslash S$. Without loss of generality, we can assume that no uncovered client receives any service, i.e., for every $j \in \bar{S}, x(j)=0$.

If $S=J$, then $x$ is an optimal cover plan, and therefore clearly a $\frac{1-r}{2-r}$ approximate solution. Assume therefore that $S \subseteq J$. First note that for every $i \in N(S)$, one of the following holds:

- $\quad$ Either there are no edges between $i$ and $\bar{S}$, or

- $x(i)=x(i, S)>(1-r) c(i)$.

To see this, assume by contradiction that there exists an $i \in N(S)$ such that there are edges between $i$ and $\bar{S}$, and $x(i) \leq(1-r) c(i)$. By the assumption, there exists at least one client $j \in \bar{S}$ such that $(i, j) \in E$. Consider the function $x^{\prime}: E \rightarrow \mathbb{R}^{+}$defined by

$$
x^{\prime}\left(i^{\prime}, j^{\prime}\right)= \begin{cases}d\left(j^{\prime}\right) & \text { if } i^{\prime}=i, j^{\prime}=j \\ x\left(i^{\prime}, j^{\prime}\right) & \text { otherwise. }\end{cases}
$$

Clearly, for every $i^{\prime} \neq i, x^{\prime}$ does not violate the capacity constraint imposed by $c\left(i^{\prime}\right)$, since by the feasibility of $x$, 
for every such $i^{\prime}, x^{\prime}(i)=x(i) \leq c(i)$. Furthermore, since $x$ was a cover-by-one plan, then so is $x^{\prime}$. Consider base station $i$. Since by the assumption $x(i) \leq(1-r) c(i)$, using the fact that the instance is $r$-restricted, we have $x^{\prime}(i)=x(i)+d\left(j^{\prime}\right) \leq c(i)$, hence the capacity constraint is satisfied for $i$ as well. Finally, note that all clients $j^{\prime} \in$ $S \cup\{j\}$ are satisfied by the cover plan $x^{\prime}$. It follows that $x^{\prime}$ is an $S \cup\{j\}$-extension of $x$, contradicting the assumption that $x$ is CBO-maximal. Using a similar argument one can show that $N(\bar{S}) \subseteq N(S)$, otherwise there is a base station in $N(\bar{S}) \backslash N(S)$ that can satisfy at least one client in $\bar{S}$, contradicting the maximality of $S$. It follows that for every $i \in N(\bar{S}), x(i)>(1-r) c(i)$.

Let $\mathrm{OPT} \subseteq J$ denote any optimal solution to the problem, assuming the cover-by-many paradigm. Note that

$$
\begin{aligned}
p(\mathrm{OPT})= & p(\mathrm{OPT} \cap S)+p(\mathrm{OPT} \cap \bar{S}) \leq p(S) \\
& +\epsilon \cdot \sum_{j \in \mathrm{OPT} \cap \bar{S}} d(j) \leq p(S)+\epsilon \cdot c(N(\bar{S})),
\end{aligned}
$$

where the last inequality follows from the feasibility of OPT.

On the other hand, by the maximality of $S$, we are guaranteed to have

$$
\begin{aligned}
d(S) & =\sum_{j \in S} d(j)=\sum_{i \in I} x(i) \geq \sum_{i \in N(\bar{S})} x(i) \\
& >\sum_{i \in N(\bar{S})}(1-r) \cdot c(i)=(1-r) \cdot c(N(\bar{S})),
\end{aligned}
$$

which in turn implies

$$
p(S)=\epsilon \cdot d(S)>\epsilon(1-r) \cdot c(N(\bar{S})) .
$$

It follows that

$$
p(\mathrm{OPT}) \leq p(S)+\frac{p(S)}{1-r}=p(S)\left(1+\frac{1}{1-r}\right)=\frac{2-r}{1-r} \cdot p(S)
$$

hence $S$ is a $\frac{1-r}{2-r}$ approximate solution w.r.t the profit function $p$, when compared to the optimal cover-bymany solution.

Theorem 4.5. Algorithm CBO-MC produces a $\frac{1-r}{2-r}$-approximate solution. Furthermore, this approximation is with regards to the optimal cover-by-many solution.

Proof. We prove by induction on the recursion that the cover plan returned from every call is a $\frac{1-r}{2-r}$-approximate solution. Note that the number of clients in every two consecutive recursive calls decreases by at least 1 , thus the recursion will terminate.

For the base case, since $J=\emptyset$, there are no clients to cover, hence $x \equiv 0$ is an optimal cover, and therefore clearly a $\frac{1-r}{2-r}$-approximate solution. For the inductive step, we have two cases to consider. First, consider the cover plan $x^{\prime}$ for $B \subseteq J \backslash\{j\}$ returned in line 6. By the induction hypothesis, $B$ is a $\frac{1-r}{2-r}$ approximate solution w.r.t. the graph $G^{\prime}=(I, J \backslash\{j\}, E \backslash E(j))$ and profit function $p$. Since $p(j)=0$, the optimal profit w.r.t the graph $G=$ $(I, J, E)$ and profit function $p$ cannot be greater than the optimal profit w.r.t the graph $G^{\prime}$ and profit function $p$. Hence, $B$ is also a $\frac{1-r}{2-r}$ approximate solution w.r.t. the graph $G=(I, J, E)$ and profit function $p$. The second case to consider is the cover plan $x^{\prime}$ for $B$ returned in line 20. By the induction hypothesis, $B$ is a $\frac{1-r}{2-r}$ approximate solution w.r.t. the graph $G=(I, J, E)$ and profit function $p_{2}$. Since for every client $j$ considered in lines 13-19, $p_{2}(j)=0$, the optimal profit w.r.t the graph $G=(I, J, E)$ and profit function $p_{2}$ cannot be greater than the optimal profit attainable from the instance returned from the recursive call. Hence, the solution returned in line 20 is a $\frac{1-r}{2-r}$ approximate solution w.r.t. the graph $G=(I, J, E)$ and profit function $p_{2}$, and so is any extension of it using clients $j$ such that $p_{2}(j)=0$. Note that for every client $j$ such that $p_{2}(j)=0$, who has a neighbor with sufficient residual capacity, $j$ is added to the cover, where exactly one base station is used to satisfy its demand. It follows that the solution returned in line 20 is a CBO-maximal solution. By Lemma 4.4 it follows that this solution is a $\frac{1-r}{2-r}$ approximate solution w.r.t. the graph $G=(I, J, E)$ and profit function $p_{1}$. Using Lemma 4.3 we conclude that the solution returned is a $\frac{1-r}{2-r}$ approximate solution w.r.t. the graph $G=(I, J, E)$ and profit function $p=p_{1}+p_{2}$, which completes the proof.

Note that the solution $x$ produced by algorithm СВО-МС is a cover-by-one plan. It therefore follows that the ratio between the optimal cover-by-one solution and the optimal cover-by-many solution is at most $\frac{1-r}{2-r}$ as well.

The running time of $\mathrm{CBO}-\mathrm{MC}$ is governed by two components: 1) in each recursive call we sort the current set of items (to either find the minimum profit or minimum ratio, in lines 4 and 9, respectively), and 2) finding the candidate base station for coverage (in line 14). This gives an overall running time of $O\left(n^{2} \log n+n m\right)$.

\subsection{A Cover-by-Many $(1-r)$-Approximation Algorithm}

We now turn to describe our second algorithm, called CBMMC, which achieves an approximation ratio of $(1-r)$ using the cover-by-many paradigm. Under CBM-MC, a client is added by first trying to exhaust the capacities of base stations which cannot contribute to uncovered clients, and then using the capacity of the remaining base stations in order to complete the cover. If such a cover cannot be produced, then the client is discarded. The pseudocode of the algorithm is given in Algorithm 2, where we use the subroutine MAXFLOW $\left(G_{f}(A, B)\right)$ to denote the computation of the maximum $s$ - $t$ flow in the flow graph $G_{f}(A, B)$ using an algorithm that finds a maximum flow, which may extend a pregiven initial feasible flow from $s$ to $t$. An example of such algorithms include algorithms based on finding augmenting paths, such as the Edmonds-Karp algorithm [12]. The reason we require such an algorithm is due to the fact that our algorithm must ensure the property of CBM maximality is maintained whenever a client is added to the cover (recall the definition of CBM maximality appearing at the end of Section 4.2). Note that by duality, given any $s$ - $t$ flow in a flow graph $G_{f}(A, B)$, it is easy to verify if a cut is a minimum cut by checking that all the edges are saturated. For concreteness and clarity, throughout our proof we assume the algorithm used is the Edmonds-Karp algorithm, and thus use augmentingpaths terminology. 
Algorithm 2. CBM-MC $(G=(I, J, E)$, demands $d$, profits $p$, capacities $c$ ).

1: $x \leftarrow \operatorname{MAXFLOW}\left(G_{f}\right)$

2: if $\{t\}$ is a MINCUT in $G_{f}$ then

3: $\quad$ return $x$

4: end if

5: if there exists a $j \in J$ such that $p(j)=0$ then

6: $\quad x \leftarrow \operatorname{CBM-MC}\left(G^{\prime}=(I, J \backslash\{j\}, E \backslash E(j)), d, p, c\right)$

7: $\quad$ return $x$

8: else

9: $\quad$ for every $j \in J$, set $\epsilon_{j}=\frac{p(j)}{d(j)}$

10: $\quad$ set $\epsilon=\min _{j} \epsilon_{j}$

11: $\quad$ for every $j \in J$, set $p_{1}(j)=\epsilon \cdot d(j)$

12: $\quad$ set $p_{2}=p-p_{1}$

13: $\quad x \leftarrow$ CBM-MC $\left(G, d, p_{2}, c\right)$

14: $\quad$ for every $j$ such that $p_{2}(j)=0$ do

15:

16:

17:

18:

19:

21

22:

23:

24:

\section{5: end if}

$$
\begin{aligned}
& S \leftarrow\left\{j^{\prime} \in J \mid x\left(j^{\prime}\right)=d\left(j^{\prime}\right)\right\} \\
& \text { set } N_{\bar{S} \backslash\{j\}}=N(J \backslash(S \cup\{j\})) \\
& \text { set } Y_{S \cup\{j\}}=I \backslash N_{\bar{S} \backslash\{j\}} \\
& y \leftarrow \operatorname{MAXFLOW}\left(G_{f}\left(Y_{S \cup\{j\}}, S \cup\{j\}\right)\right) \\
& \triangleright \text { See Fig. 2a }
\end{aligned}
$$

$z \leftarrow \operatorname{MAXFLOW}\left(G_{f}(I, S \cup\{j\})\right)$, starting from the initial feasible flow $y$. $\quad \triangleright$ See Fig. $2 \mathrm{~b}$

if $\{t\}$ is a MINCUT in $G_{f}(I, S \cup\{j\})$ then

$$
x \leftarrow z
$$

$$
\text { end if }
$$

end for

return $x$

Given a cover plan $x$ for $S \subseteq J$, let $\bar{S}=J \backslash S$, and consider $I$ as partitioned into two sets: $N_{\bar{S}}=N(\bar{S})$, and $Y_{S}=I \backslash N_{\bar{S}}$. Note that by definition, for every $j \in \bar{S}$ and $i \in Y_{S},(i, j) \notin E$. The following lemma provides a necessary and sufficient condition for covering a set of clients.

Lemma 4.6. For any instance of $r-A o N D M$ over a graph $G=(I, J, E)$, and any $A \subseteq I$ and $B \subseteq J,\{t\}$ is a minimum $s$-t cut in the flow-graph $G_{f}(A, B)$ if and only if $A$ can cover all clients in $B$.

Proof. Let $x$ be a maximum $s$ - $t$ flow in $G_{f}(A, B)$. By duality, the value of the maximum $s$ - $t$ flow is the same as the capacity of the minimum $s$ - $t$ cut, and the edges of any such cut are all saturated by any maximum flow. Assume $\{t\}$ is a minimum s-t cut in $G_{f}(A, B)$, and assume by contradiction that there is client $j \in B$ which is not fully covered. It follows that $\sum_{i:(i, j) \in E} x(i, j)<d(j)$. By flow conservation, we have $x(j, t)=\sum_{i:(i, j) \in E} x(i, j)$, which implies $x(j, t)<d(j)$. Since $(j, t)$ is an edge in the minimum $s$ - $t$ cut $\{t\}$, and its capacity is $d(j)$, this contradicts the fact that all such edges are saturated by the maximum flow $x$. Assume now $\{t\}$ is not a minimum $s$ - $t$ cut, and assume by contradiction that all clients can be covered. By flow conservation it follows that for every $j \in J, \quad \sum_{i:(i, j) \in E} x(i, j)=d(j)=x(j, t)$, which by summing over all $j \in J$ implies that the value of the flow equals the capacity of the cut $\{t\}$. By duality, this implies that $\{t\}$ is a minimum $s-t$ cut, contradicting our assumption.

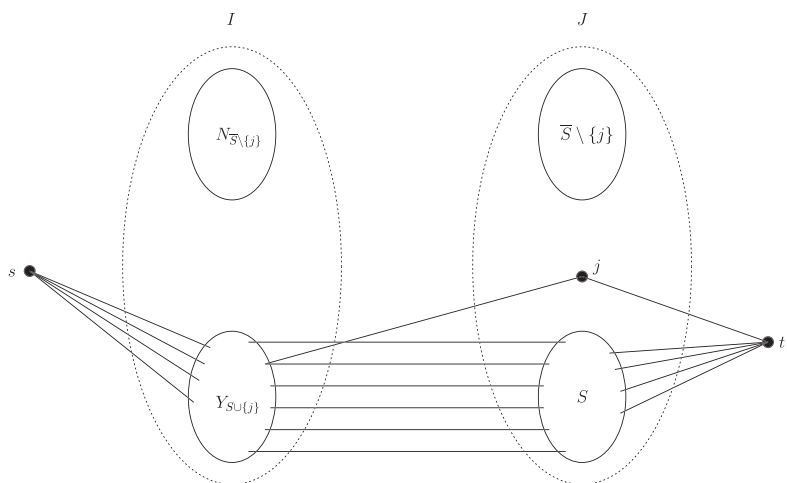

(a) Topology of $G_{f}\left(Y_{S \cup\{j\}}, S \cup\{j\}\right)$.

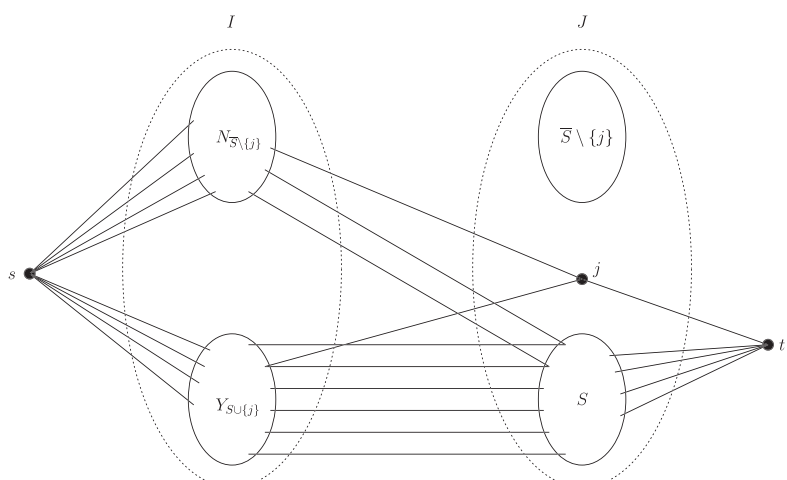

(b) Topology of $G_{f}(I, S \cup\{j\})$.

Fig. 2. Outline of the flow graphs used in CBM-MC.

Lemma 4.6 admits a method for finding a rearrangement-maximal cover plan, as shown in the following lemma:

Lemma 4.7. Given any instance of $r-A o N D M$ over a graph $G=(I, J, E)$, any cover plan $x$ for $S \subseteq J$, and a client $j \in J \backslash S$, the task of finding an $S \cup\{j\}$-rearrangement of $x$, if one exists, can be done in polynomial time.

Proof. In order to find a rearrangement of $x$, consider the flow graph $G^{\prime}=G_{f}(I, S \cup\{j\})$, and let $y$ be a maximum flow in this graph. If $\{t\}$ is a minimum cut in $G^{\prime}$, then by Lemma 4.6, $y$ is a cover plan for $S \cup\{j\}$, and hence it is an $S \cup\{j\}$-rearrangement. If $\{t\}$ is not a minimum cut in $G_{f}^{\prime}$, then its capacity is strictly greater than the maximum flow, and therefore not all clients in $S \cup\{j\}$ can be satisfied. Verifying whether or not $\gamma(t)=y(t)$ can clearly be done in polynomial time. This is illustrated in Fig. 2. $\square$

The following is an immediate corollary of Lemma 4.7.

Corollary 4.8. Given any instance to $r-A o N D M$ over a graph $G=(I, J, E)$, any cover plan $x$ for $S \subseteq J$, and a client $j \in J \backslash S$, the task of finding a rearrangement of $x$ which is rearrangement-maximal can be done in polynomial time.

Proof. By iteratively applying Lemma 4.7, we are guaranteed to obtain a rearrangement-maximal cover plan.

The following lemmas describe the correlation between the maximum flow in $G_{f}$, and the maximum flow in flow graphs of the form $G_{f}\left(Y_{S}, S\right)$, for sets $S$ which have a cover plan. 
Lemma 4.9. Assume $S \subseteq J$ has some cover plan. Then, there exists a maximum flow $x$ in $G_{f}$ such that $x\left(Y_{S}, S\right)=$ $\operatorname{MAXFLOW}\left(G_{f}\left(Y_{S}, S\right)\right)$. Furthermore, such a flow can be found in polynomial time.

Proof. Let $y=\operatorname{MAXFLOW}\left(G_{f}\left(Y_{S}, S\right)\right)$. Clearly, $y$ is a feasible flow in $G_{f}$ as well. Consider the Edmonds-Karp Algorithm MAXFLOW, see [12] for details) for finding a maximum flow, executed on graph $G_{f}$, starting from the initial feasible flow $y$. We show that for every augmentation path found by MAXFLOW, after increasing the flow along this path and obtaining some flow $y^{\prime}, y^{\prime}\left(s, Y_{S}\right) \geq y\left(s, Y_{S}\right)$.

First note that we can assume that all the augmentation paths used by the MAXFLOW algorithm are simple paths. Furthermore, note that by the fact that any augmentation path is simple, we obtain that for every flow $y^{\prime}$ obtained during executing the MAXFLOW algorithm, and for every $i \in Y_{S}, y(s, i) \leq y^{\prime}(s, i)$, since such flow can only decrease if the algorithm uses a path $p$ such that $(i, s) \in p$, which implies that $p$ is not a simple path.

Since for every feasible flow $z$ we have $z\left(Y_{S}, S\right)=$ $z\left(s, Y_{S}\right)$ (by flow conservation, and using the fact that there are no edges between $Y_{S}$ and $\bar{S}$ ), we can conclude that during the entire execution of the MAXFLOW algorithm, the flow $y^{\prime}$ resulting in augmenting any path $p$ satisfies $y^{\prime}\left(s, Y_{S}\right) \geq y\left(s, Y_{S}\right)$. On the other hand, note that given any maximum flow in $G_{f}$, if we consider its flow path decomposition, then the set of paths using edges between $Y_{S}$ and $S$ also constitutes a flow in $H_{S}$ (due to the unidirectionality of edges between $N_{\bar{S}}$ and $S$ in $G_{f}$ ). Hence, these paths cannot support a flow whose value is greater than MAXFLOW $\left(G_{f}\left(Y_{S}, S\right)\right)$.

Finally note that MAXFLOW produces a maximum flow in $G_{f}$ in polynomial time, which completes the proof of the lemma.

The above lemma gives rise to the following corollary:

Corollary 4.10. If there exists a rearrangement-maximal cover plan $y$ for $S \subseteq J$, then there exists a CBM-maximal cover plan $x$ for S. Furthermore, such a cover plan can be found in polynomial time.

Proof. Using a similar argument as the one used in Lemma 4.9 , by running MAXFLOW with an initial feasible flow $z=\operatorname{MAXFLOW}\left(G_{f}\left(Y_{S}, S\right)\right)$, we are guaranteed to produce a cover plan for $S$ (by the existence of $y, S$ can be covered by $I$ ). Furthermore, this cover must also be CBM-maximal. Note that such a cover plan can be found in polynomial time by the same arguments as the ones used in Lemma 4.9.

The following lemma shows a bound on the value of any maximum flow in $G_{f}$.

Lemma 4.11. Given any $S \subseteq J$, if $S$ has a CBM-maximal cover plan, then $\operatorname{MAXFLOW}\left(G_{f}\right) \leq \operatorname{MAXFLOW}\left(G_{f}\left(Y_{S}, S\right)\right)+$ $c\left(N_{\bar{S}}\right)$.

Proof. Let $y$ be a CBM-maximal cover plan for $S$, and consider a partition of $y$ into two types of flow paths, each consisting of three edges:

- $T_{1}=\left\{p=(s, i, j, t) \mid\right.$ such that $\left.i \in Y_{S}\right\}$.

- $T_{2}=\left\{p=(s, i, j, t) \mid\right.$ such that $\left.i \in N_{\bar{S}}\right\}$.
Note that such a packing exists, by the directionality of the edges in $G_{f}{ }^{2}$ If we denote the flow along a flow path $p$ by $x(p)$, then clearly

$$
\sum_{p \in T_{1}} x(p) \leq \operatorname{MAXFLOW}\left(G_{f}\left(Y_{S}, S\right)\right),
$$

since all paths in $T_{1}$ are paths in $G_{f}\left(Y_{S}, S\right)$, and therefore cannot support a flow greater than MAXFLOW $\left(G_{f}\left(Y_{S}, S\right)\right)$. On the other hand,

$$
\sum_{p \in T_{2}} x(p) \leq c\left(s, N_{\bar{S}}\right)=c\left(N_{\bar{S}}\right),
$$

since all these paths use edges in the cut $\left(s, N_{\bar{S}}\right)$. It therefore follows that:

$$
\operatorname{MAXFLOW}\left(G_{f}\right) \leq \operatorname{MAXFLOW}\left(G_{f}\left(Y_{S}, S\right)\right)+c\left(N_{\bar{S}}\right) .
$$

We can now continue in the same way as we did with the simpler algorithm, where CBM-maximality replaces CBOmaximality.

Lemma 4.12. Consider any instance of the $r$-AoNDM problem such that for every client $j, p(j)=\epsilon \cdot d(j)$, for some constant $\epsilon$. Any cover plan $x$ for $S \subseteq J$ which is CBM-maximal is a $(1-r)$-approximate solution w.r.t. profit function $p$.

Proof. Let $x$ be any cover plan for $S \subseteq J$ which is CBMmaximal. If $S=J$, then $x$ is an optimal cover plan, and therefore clearly a $(1-r)$ approximate solution. Assume $S \subseteq J$. Note that by maximality of

$$
x, x\left(Y_{S}, S\right)=\operatorname{MAXFLOW}\left(G_{f}\left(Y_{S}, S\right)\right),
$$

and since $S \subseteq J, x\left(N_{\bar{S}}, S\right)>(1-r) c\left(N_{\bar{S}}\right)$, i.e., $c\left(N_{\bar{S}}\right)<$ $\frac{x\left(N_{\bar{S}}, S\right)}{1-r}$. By the fact that $x$ is a cover plan for $S$, we have $p(S)=\epsilon d(S)=\epsilon\left(x\left(N_{\bar{S}}, S\right)+x\left(Y_{S}, S\right)\right)$, since $N_{\bar{S}}, Y_{S}$ are a partition of $I$.

Let $\mathrm{OPT} \subseteq J$ denote any optimal solution to the problem. We wish to bound the value of $p(\mathrm{OPT})$. Clearly, for any maximum $s$ - $t$ flow $y$ in $G_{f}, d(\mathrm{OPT}) \leq y(s)$, since any cover plan for OPT induces a feasible flow in $G_{f}$. Combining the above with Lemma 4.11 we obtain that for any maximum $s$ - $t$ flow $y$ in $G_{f}$,

$$
\begin{aligned}
d(\mathrm{OPT}) \leq & y(s) \\
\leq & \operatorname{MAXFLOW}\left(G_{f}\left(Y_{S}, S\right)\right)+c\left(N_{\bar{S}}\right) \\
& <x\left(Y_{S}, S\right)+\frac{x\left(N_{\bar{S}}, S\right)}{1-r} \\
= & \frac{1}{1-r}\left((1-r) \cdot x\left(Y_{S}, S\right)+x\left(N_{\bar{S}}, S\right)\right) \\
\leq & \frac{1}{1-r}\left(x\left(Y_{S}, S\right)+x\left(N_{\bar{S}}, S\right)\right) \\
= & \frac{1}{1-r} d(S) .
\end{aligned}
$$

By the definition of $p$ we obtain that $p(S)>(1-r)$. $p(\mathrm{OPT})$, which completes the proof.

2. Note that these are not augmentation paths used in computing the maximum flow by MAXFLOW. These paths are part of an actual path decomposition of the maximum flow. 
Theorem 4.13. Algorithm CBM-MC produces a $(1-r)$ approximate solution.

Proof. We prove by induction on the recursion that the cover plan returned from every call is a $(1-r)$-approximate solution, similarly to the proof of Theorem 4.5.

For the base case, if $\{t\}$ is minimum cut, then by Lemma 4.6 all the clients can be covered, hence $x$ is an optimal cover plan, and therefore clearly a $(1-r)$ approximate solution. For the inductive step, we have two cases to consider. First, consider the cover plan $x^{\prime}$ for $B \subseteq J \backslash\{j\}$ returned in line 7 . By the induction hypothesis, $B$ is a $(1-r)$-approximate solution w.r.t. the graph $G^{\prime}=(I, J \backslash\{j\}, E \backslash E(j))$ and profit function $p$. Since $p(j)=0$, the optimal profit w.r.t the graph $G=(I, J, E)$ and profit function $p$ cannot be greater than the optimal profit w.r.t the graph $G^{\prime}$ and profit function $p$. Hence, $B$ is also a $(1-r)$-approximate solution w.r.t. the graph $G=(I, J, E)$ and profit function $p$. The second case to consider is the cover plan $x^{\prime}$ for $B$ returned in line 24. By the induction hypothesis, $B$ is a $(1-r)$ approximate solution w.r.t. the graph $G=(I, J, E)$ and profit function $p_{2}$. Since for every client $j$ considered in lines 13-19, $p_{2}(j)=0$, the optimal profit w.r.t the graph $G=(I, J, E)$ and profit function $p_{2}$ cannot be greater than the optimal profit attainable from the instance returned from the recursive call. Hence, the solution returned in line 20 is a $(1-r)$-approximate solution w.r.t. the graph $G=$ $(I, J, E)$ and profit function $p_{2}$, and so is any superset of this solution produced by adding any of the clients for which $p_{2}(j)=0$.

Note that by Lemma 4.7, for every client $j \notin S$ such that $p_{2}(j)=0$, lines 19 and 20 compute an $S \cup\{j\}$ rearrangement of the current cover plan, if such a rearrangement exists. Hence, the resulting solution returned in line 24 is a rearrangement-maximal solution. In addition, by lines 18-19, the cover plan $x^{\prime}$ computed in every iteration also satisfies that $x\left(Y_{S}, S\right)$ is a maximum flow in the flow graph $G_{f}\left(Y_{S}, S\right)$. It therefore follows that the cover plan returned in line 24 is also CBM-maximal. By Lemma 4.12 it follows that this solution is a $(1-r)$ approximate solution w.r.t. the graph $G=(I, J, E)$ and profit function $p_{1}$. Using Lemma 4.3 we conclude that the solution returned is a $(1-r)$ approximate solution w.r.t. the graph $G=(I, J, E)$ and profit function $p=p_{1}+p_{2}$, which completes the proof.

The running time of CBM-MC is governed by two components: 1 ) in each recursive call we sort the current set of items (to either find the minimum profit or minimum ratio, in lines 4 and 9, respectively), and 2) computing the max flow on lines 18 and 19. If we denote by $F$ the running time of the max-flow algorithm used in lines 18 and 19, we obtain an overall running time of $O\left(n^{2} \log n+n F\right)$. In case we use the Edmonds-Karp maxflow algorithm, which finds a maximum flow in a graph $G=(V, E)$ in time $O\left(|V||E|^{2}\right)$, we obtain an overall running time of $O\left((n+m) n^{3} m^{2}\right)$.

\section{Simulation Results}

In the previous sections, we proposed two different algorithms for a new global mechanism for cell selection in $4 \mathrm{G}$ cellular networks. The main difference between these two algorithms is the way the demand of a mobile client is satisfied. In the CBO-MC Algorithm (Section 4.3) at most one base station satisfies the demand of any given mobile station while the CBM-MC Algorithm (Section 4.4) allows satisfaction of the demand simultaneously by more than one base station.

In order to study the expected performance of the proposed global cell selection algorithms with respect to the current local mobile SNR-based protocol we conducted several simulations over high-loaded, capacity constrained, 4G-like networks. A secondary goal of these simulations was to study the "benefit" of using the new ability, as made possible by the evolving standards of IEEE $802.16 \mathrm{~m}$ and LTE-Advanced, of a mobile station to be serviced simultaneously by more than one base station.

\subsection{Methodology}

We considered a network consisting of an $n \times n$-grid of clients' locations (demand points, each considered as a single client, or bin). Each client has a service request for either voice or data service. The demand of a voice and data client is defined as 1 and 25, respectively. ${ }^{3}$ Under this ratio between the demand of data and voice clients, the number of the data clients was chosen so that the overall voice volume is 20 percent of the network's traffic. ${ }^{4}$ The locations for each type of client was uniformly and randomly selected over the grid. The profit for satisfying the demand of a voice client was defined as 1 , while satisfaction of a data client is credited with a profit that is proportional to its demand (i.e., 25 units of profit).

We maintain microcells and picocells in our network. Since we implemented the restricted version of AoNDM, the demand of every client must be less than or equal to an $r$-fraction of the capacity of any base station service this client. Therefore, the capacity of a picocell was taken to be about $25 / r$, for any given value of $0<r<1$. To simulate high-loaded networks we assumed that the total sum of (client) demands equals the sum of (base station) capacities in the network. The ratio between the number of picocells and microcells was defined to be $\lambda$ while this factor was also selected as the ratio between the corresponding radiuses and capacities of microcells and picocells. By taking $\lambda=5$, we can now derive the appropriate number of microcells and picocells. The locations for each type of base station was uniformly and randomly selected over the grid and clients were associated with (omnidirectional) base stations according to their distance from each of the centers.

In each of the following three sets of simulations we measured the ratio between the total profit achieved by each of the three algorithms and the total profit of all connected clients, i.e., clients that are within service range of some base station. As AoNDM is NP-hard, the maximum possible profit is hard to calculate, and we consider the total profit of all connected clients as an upper bound on the optimal solution.

3. The bit rate for voice applications is $64 \mathrm{Kbps}$ and the downlink rate for data application is approximately $2 \mathrm{Mbps}$ in HSDPA. This gives a ratio of 25-30 between the demand of voice and data clients.

4. To be precise, if $n_{v}$ and $n_{d}$ are the number of voice and data clients, respectively, and $d_{v}$ and $d_{d}$ are the corresponding demands, then the following are satisfies for an overall voice volume of $\gamma$ of the network's traffic: $\frac{d_{v} \cdot n_{v}}{d_{v} \cdot n_{v}+d_{d} \cdot n_{d}}=\gamma, n_{d}=n^{2}-n_{v}$, and $n_{v}=\left\lfloor\frac{\gamma \cdot d_{d} \cdot n^{2}}{\left(d_{d}-d_{v}\right) \cdot \gamma+1}\right\rfloor$. In our case $\gamma=0.2$. 


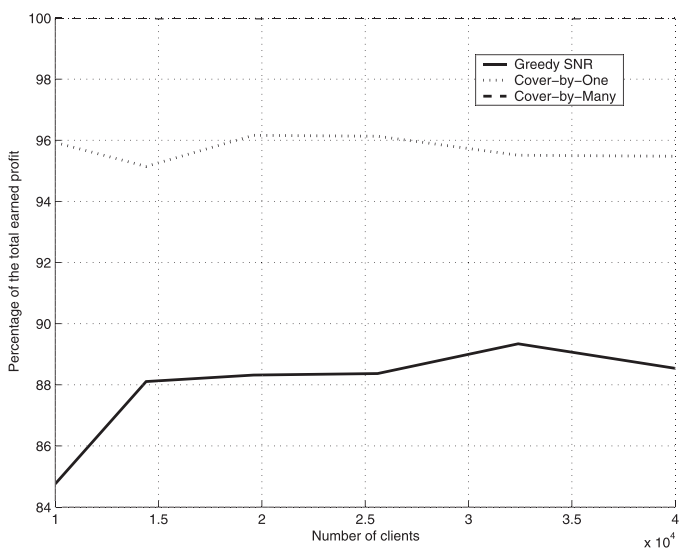

Fig. 3. Expected profit as a function of the number of clients, $r=0.05$.

\subsection{Results}

In the first set of simulations, we study the performance of the three algorithms over various network sizes (10K to $40 \mathrm{~K})$ and different values of $r$ (0.05 to 0.3). Typical results are shown in Figs. 3, 4, and 5, where the upper, middle and the lower curves correspond to the cover-by-many algorithm, cover-by-one algorithm, and the greedy-best detected-SNR algorithm, respectively. In each of the three scenarios, our results show that the cover-by-many algorithm is better than the cover-by-one algorithm by 5 percent (for $r=0.05$ ) to 11 percent (for $r=0.3$ ). An improvement of at least 10 percent (and up to 20 percent) was achieved by the cover-by-many algorithm in comparison with the greedy-best detected-SNR algorithm. The results show that the performances of all three algorithm are nearly independent of the size of the network. Moreover, due to the existence of the simultaneous coverage in the third algorithm, when $r$ increases the "distance" between the performance of the cover-by-many algorithm and the other two algorithms also increases in a significant fashion. This shows that when there exist mobile clients with demands that are relatively close to the capacity of the servicing cell (e.g., in case of picocells) allowing satisfaction of a client by more than one base station is crucial in order to maintain high utilization of the network capacities.

The second set of simulations investigates the level of profit achieved by the three algorithms when the value of $r$ varies (from $r=0.01$ to $r=0.5$ ). We fixed a network of

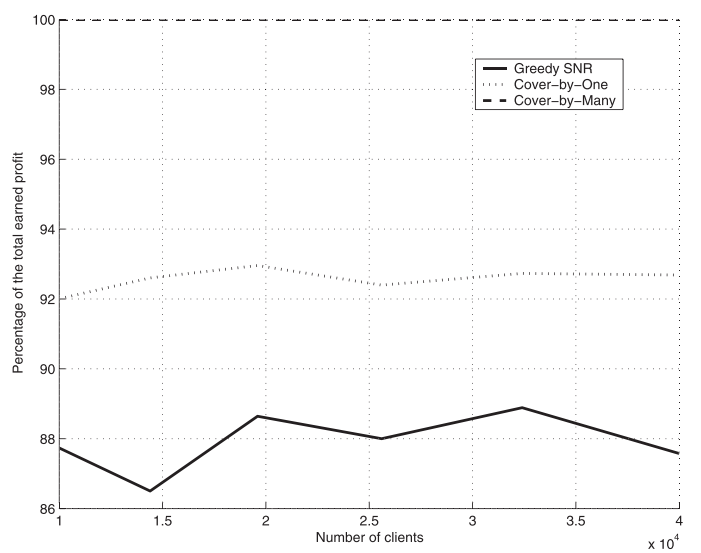

Fig. 4. Expected profit as a function of the number of clients, $r=0.1$.

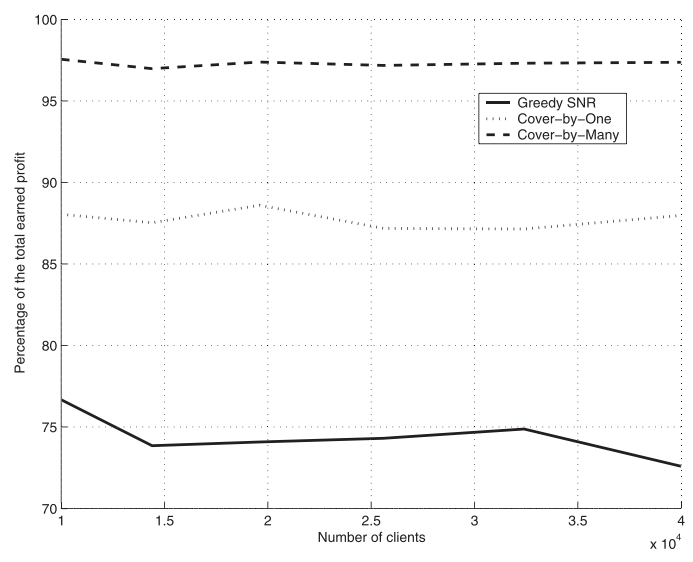

Fig. 5. Expected profit as a function of the number of clients, $r=0.3$.

15,129 clients (i.e., a grid of $123 \times 123$ ) with a number of picocells and microcells as explained above. Focusing on the relative fraction of the demand of a client with respect to the capacity of any serviced base station, the results show (Fig. 6) that when this fraction increases the ability to reach a higher percentage of the total possible profit decreases. As shown in Fig. 6, all three algorithms exhibit the same behavior. The performance of the cover-by-many algorithm (upper curve) decreases from 100 to 89 percent when $r$ increases from 0.01 to 0.5 . The cover-by-one algorithm decreases by 21 percent (from 100 percent in $r=0.01$ to 79.5 percent in $r=0.5$ ), and the greedy-best detected-SNR algorithm (lower curve) exhibited a decrease of 30 percent (from 89 to 59 percent). The third set of simulations examines the level of profit obtained by the three algorithms when the available capacity increases. We fixed a network of 15,129 clients, where each client has a demand (of any service) that is at most a fraction of $1 / 4$ $(r=0.25)$ of the capacity of each of the servicing base stations. In this study, the number of picocells as well as microcells was increased by $j$ times their basic number, $j=1,1.5,2, \ldots, 5$, where the basic numbers are the same as the ones computed in the first set of simulations (65 microcells and 327 picocells). Note that for $j>1$, the total capacity is higher than the total demand of clients. As one might expect (see Fig. 7), when there is a larger number of base stations the performance of the three algorithms can only improve. The greedy-best detected-SNR algorithm (lower curve) achieve an improvement of up to 8 percent

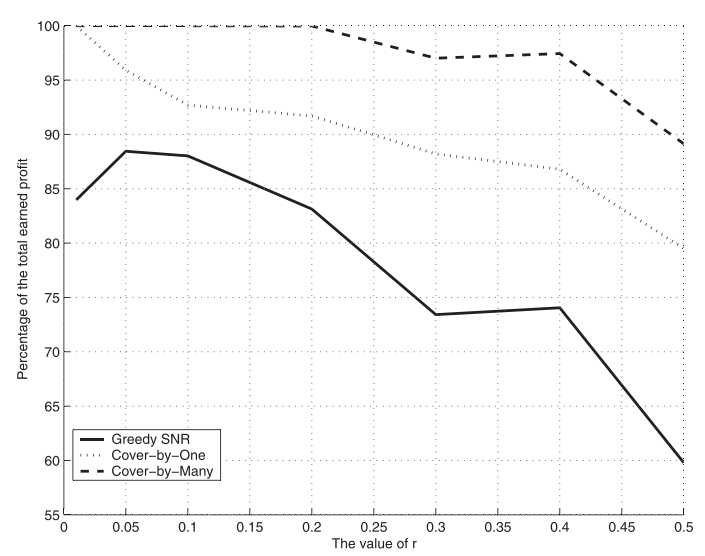

Fig. 6. Expected profit as a function of $r(n=15,129)$. 


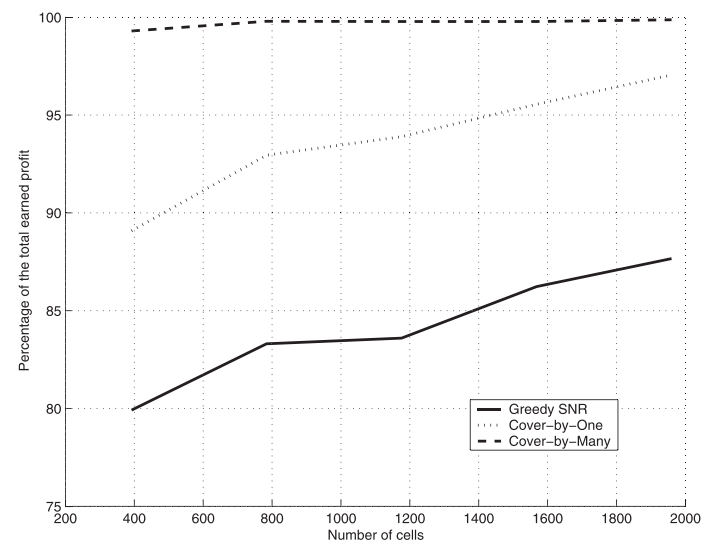

Fig. 7. Expected profit as a function of available capacity $(r=0.25$, $n=15,129$ ).

(from 79 to 87 percent) when the number of base station grows from 392 to 1,960. The cover-by-one algorithm (in the middle) achieves an improvement of up to 8 percent (from 89 to 97 percent), and the cover-by-many algorithm (upper curve) is nearly constant (around 99 percent) in its ability to satisfy clients.

Finally, the worst case running time of each of the algorithms, for all cases, was approximately 4 minutes for the case of $n=40,000, r=0.25$, on a Pentium M machine, $1.4 \mathrm{GHz}$, and $256 \mathrm{Mb}$ of RAM.

\section{Conclusions}

In this paper, we present a rigorous study of a new approach for cell selection in fourth generation cellular networks. Unlike the current cell selection protocol, our proposed mechanism is global, has a performance guarantee, and addresses many of the anticipated $4 \mathrm{G}$ technologies. We show that even though AoNDM is hard to approximate to within a reasonable factor, we can still cover all practical scenarios by adopting the assumption that every mobile station has a traffic demand that is relatively smaller than the capacity of any base station that is able to participate in its coverage. We give two approximation algorithms for this problem. The first is a $\frac{1-r}{2-r}$-approximation algorithm for the case where each mobile station can be covered by exactly one base station (cover-by-one). The second is a slower, delicate refinement of the first algorithm, guaranteeing a $(1-r)$-approximate solution, that adopt the new IEEE $802.16 \mathrm{~m}$ and LTE-Advanced possibility of simultaneously servicing a mobile clients by more than one base station (cover-by-many). We compare between global mechanisms that are based on our approximation algorithms and a local procedure performed by the current best-SNR greedy cell selection protocol. We show that when clients of very high bandwidth demand, relatively to the base station's capacity, exist, the use of multiple base station to satisfy the demand of a mobile station can maintain a level of at least 97 percent of the possible coverage- 20 percent better coverage than the current best-SNR greedy cell selection method. In addition to $4 \mathrm{G}$ networks, such relevant scenarios may be found in spread areas where there are several very small populated areas and "standard" infrastructure is not costeffective. In these areas, coverage can be achieved using several WiMAX-cells and situations where such cells are overloaded may be common. Our scheme for cell selection can be used in order to allow a better utilization of these coverage solutions.

There are several interesting problems that arise from this work. The first and most important aspect is to devise an online, distributed, algorithm for doing cell selection. Such algorithms will undoubtedly make extensive use of the network initiated handoffs, which are available, e.g., under the specification of IEEE $802.16 \mathrm{~m}$ and LTE-Advanced. Solving such problems should also be extended to consider user mobility, and online performance for heterogeneous users. In this respect is should be noted that for real-life cellular layouts, one might expect finding restricted inputs to the problem of cell-selection. For such restricted topologies (e.g., determined by power considerations, or geometrical restrictions), it is possible better approximation can be obtained. Furthermore, we expect that good online, localcontrol, algorithms might also be developed for such restricted scenarios, demonstrating good performance. Our results and algorithms may serve as a benchmark for evaluating the performance of such algorithm. Our work also gives rise to various scheduling issues, that are related to the implementation of the cover-by-many paradigm. Such questions should address the microlevel scheduling decisions made by the base stations, as opposed to the macrolevel association that is the focus of the current paper. Another interesting question is determining the complexity of various restricted versions of the AoNDM problem. A primary question in this respect is whether or not there exists a PTAS for the $r$-AoNDM problem, and under which conditions. We note that by our reduction, the general case is proven to be hard to approximate for instances in which the demand of every client is strictly greater than the capacity of the base stations which can contribute to its coverage. On the other hand, the work of [28] implies a constant approximation if every station has sufficient capacity to cover any single user within its range. Abusing our notation, it is unclear whether this phase transition occurs in $r=1$, or is there some $r>1$ for which the $r$-AoNDM problem still adheres to good approximation algorithms.

\section{ACKNOWLEDGMENTS}

The authors would like to thank the anonymous referees for their very useful comments that helped to improve the manuscript. This research was partially supported by REMON - Israel 4G Mobile Consortium, sponsored by Magnet Program of the Chief Scientist Office in the Ministry of Industry, Trade and Labor of Israel. A Preliminary version of this work appeared in the Proceedings of IEEE INFOCOM 2008, The 27th Annual Joint Conference of the IEEE Computer and Communications Societies, pp. 700-708, 2008.

\section{REFERENCES}

[1] Requirements for Further Advancements for Evolved Universal Terrestrial Radio Access (E-UTRA) (LTE-Advanced), 3GPP TR 36.913, 2009.

[2] IEEE Standard 802.16m/D4, Section 16.5, IEEE, 2010.

[3] D. Amzallag, R. Engelberg, J. Naor, and D. Raz, "Cell Planning of 4G Cellular Networks," Technical Report CS-2008-04, Computer Science Dept., Technion - Israel Inst. of Technology, 2008.

[4] D. Amzallag, M. Livschitz, J. Naor, and D. Raz, "Cell Planning of 4G Cellular Networks: Algorithmic Techniques, and Results," Proc. IEE Sixth Int'l Conf. 3G and Beyond, pp. 501-506, 2005. 
[5] D. Amzallag, J. Naor, and D. Raz, "Coping with Interference: From Maximum Coverage to Planning Cellular Networks," Proc. Fourth Workshop Approximation and Online Algorithms (WAOA), pp. 29-42, 2006.

[6] M. Andrews and L. Zhang, "Logarithmic Hardness of the Undirected Edge-Disjoint Paths Problem," J. ACM, vol. 53, no. 5, pp. 745-761, 2006.

[7] R. Bar-Yehuda, "One for the Price of Two: A Unified Approach for Approximating Covering Problems," Algorithmica, vol. 27, no. 2, pp. 131-144, 2000.

[8] C. Chekuri and S. Khanna, "A Polynomial Time Approximation Scheme for the Multiple Knapsack Problem," SIAM J. Computing, vol. 35, no. 3, pp. 713-728, 2005.

[9] C. Chekuri, S. Khanna, and F.B. Shepherd, "The All-or-Nothing Multicommodity Flow Problem," Proc. 36th Ann. ACM Symp. Theory of Computing (STOC), pp. 156-165, 2004.

[10] C. Chekuri, S. Khanna, and F.B. Shepherd, "Multicommodity Flow, Well-Linked Terminals, and Routing Problems," Proc. 37th Ann. ACM Symp. Theory of Computing (STOC), pp. 183-192, 2005.

[11] R. Cohen, L. Katzir, and D. Raz, "An Efficient Approximation for the Generalized Assignment Problem," Information Processing Letters, vol. 100, no. 4, pp. 162-166, 2006.

[12] J. Edmonds and R.M. Karp, "Theoretical Improvements in Algorithmic Efficiency for Network Flow Problems," J. ACM, vol. 19, pp. 248-264, 1972.

[13] L. Fleischer, M.X. Goemans, V.S. Mirrokni, and M. Sviridenko, "Tight Approximation Algorithms for Maximum General Assignment Problems," Proc. 17th ACM-SIAM Symp. Discrete Algorithms (SODA), pp. 611-620, 2006.

[14] S.V. Hanly, "An Algorithm for Combined Cell-Site Selection and Power Control to Maximize Cellular Spread Spectrum Capacity," IEEE J. Selected Areas in Comm., vol. 13, no. 7, pp. 1332-1340, Sept. 1995.

[15] J. Håstad, "Clique is Hard to Approximate within $n^{1-\epsilon,}$ " Acta Math., vol. 182, pp. 105-142, 1999.

[16] R. Irmer, H.-P. Mayer, A. Weber, V. Braun, M. Schmidt, M. Ohm, N. Ahr, A. Zoch, C. Jandura, P. Marsch, and G. Fettweis, "Multisite Field Trial for LTE and Advanced Concepts," IEEE Comm. Magazine, vol. 47, no. 2, pp. 92-98, Feb. 2009.

[17] B.-H. Kim, J. Yun, Y. Hur, C. So-In, R. Jain, and A.-K. Al Tamimi, "Capacity Estimation and TCP Performance Enhancement over Mobile WiMAX Network," IEEE Comm. Magazine, vol. 47, no. 6, pp. 132-141, June 2009.

[18] C.A. Mandal, P.P. Chakrabarti, and S. Ghose, "Complexity of Fragmentable Object Bin Packing and an Application," Computers and Math. with Applications, vol. 35, pp. 91-97, June 1998.

[19] R. Mathar and M. Schmeink, "Integrated Optimzal Cell Site Selection and Frequency Allocation for Cellular Radio Networks," Telecomm. Systems, vol. 21, pp. 339-347, 2002.

[20] T. Miki, T. Ohya, H. Yoshino, and N. Umeda, "The Overview of the Fourth Generation Mobile Communication System," Proc. Fifth Int'l Conf. Information, Comm. and Signal Processing (ICICS), pp. 1551-1555, 2005.

[21] N. Naaman and R. Rom, "Packet Scheduling with Fragmentation," Proc. IEEE INFOCOM, pp. 427-436, 2002.

[22] Understanding UMTS Radio Network: Modelling, Planning and Automated Optimisation, Maciej J. Nawrocki, Mischa Dohler, and A. Hamid Aghvami, eds. John Wiley \& Sons, Ltd., 2006.

[23] "Beyond 4G: Radio Evolution for the Gigabit Experience," white paper, Nokia Siemens Networks, http://www.nokiasiemens networks.com/portfolio/products/mobile-broadband, 2011.

[24] Z. Nutov, I. Binyaminy, and R. Yuster, "A (1-1/e)-Approximation Algorithm for the Generalized Assignment Problem," Operations Research Letters, vol. 34, no. 3, pp. 283-288, 2006.

[25] B. Patt-Shamir, D. Rawitz, and G. Scalosub, "Distributed Approximation of Cellular Coverage," Proc. 12th Int'l Conf. Principles of Distributed Systems (OPODIS), pp. 331-345, 2008.

[26] A. Sang, X. Wang, M. Madihian, and R.D. Gitlin, "A Load-Aware Handoff and Cell-Site Selection Scheme in Multi-Cell Packet Data Systems." Proc. 47th IEEE Global Comm. Conf. (GlobeCom), vol. 6, pp. 3931-3936, 2004.

[27] A. Sang, X. Wang, M. Madihian, and R.D. Gitlin, "Coordinated Load Balancing, Handoff/Cell-Site Selection, and Scheduling in Multi-Cell Packet Data Systems," Wireless Networks, vol. 14, no. 1, pp. 103-120, 2008.
[28] D. Shmoys and E. Tardos, "An Approximation Algorithm for the Generalized Assignment Problem," Math. Programming, vol. 62, no. 3, pp. 461-474, 1993.

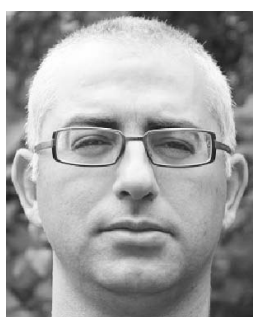

David Amzallag received the $\mathrm{PhD}$ degree in computer science from the Technion, Israel, specializing in coping with computationally hard optimization problems. As a chief technology officer and an executive vice president at Amdocs, he is responsible for planning and developing Amdocs' technology strategy and vision, as well as the architecture and roadmap of the entire product portfolio. Prior to Amdocs, he served as the chief scientist of the 21st Century Network (21CN) of British Telecom (BT), where he was responsible for designing, planning, and optimizing solutions in BT networks, architectures, and infrastructures in more than 100 countries and 250 networks worldwide. In this role, he was responsible for the development, delivery, and business transformation of BT's entire vision in these areas. He has more than 17 years of experience in leading, developing, and transforming technological strategies, technological innovation, and advanced optimization solutions in academia, start-up companies, and corporations worldwide. He has published papers and book chapters, and presented at many academic and commercial conferences.

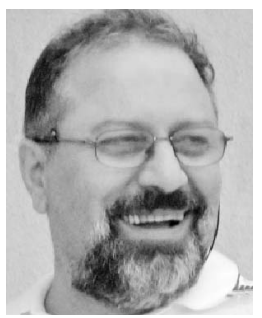

Reuven Bar-Yehuda received the BA, MSc, and DSc degrees in computer science from the Technion - Israel Institute of Technology, Haifa, in 1978, 1980, and 1983, respectively. He is currently an associate professor of computer science at the Technion. From 1984 to 1986, he was a visiting assistant professor in the Computer Science Department at Duke University. From 1992 to 1993, he was a visiting assistant professor in the Computer Science Department at The State University of New York at Buffalo. His research is in combinatorial optimization, including approximation algorithms for NPhard problems, algorithms in combinatorial geometry, distributed algorithms, theoretical aspects of radio communication, and VLSI design.

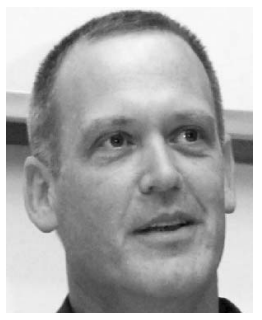

Danny Raz received the doctoral degree from the Weizmann Institute of Science, Israel, in 1995. From 1995 to 1997, he was a postdoctoral fellow at the International Computer Science Institute (ICSI), Berkeley, California, and a visiting lecturer at the University of California, Berkeley. Between 1997 and 2001, he was a member of the technical staff at the Networking Research Laboratory at Bell Labs, Lucent Technologies. In October 2000, he joined the faculty of the Computer Science Department at the Technion, Israel. He served as the general chair of OpenArch 2000, TPC cochair of MMNS 2007, and TPC cochair of IM2009, and was an editor of the IEEE/ACM Transactions on Networking (ToN) and of the Journal of Communications and Networks. His primary research interest is the theory and application of management related problems in IP networks and cloud computing.

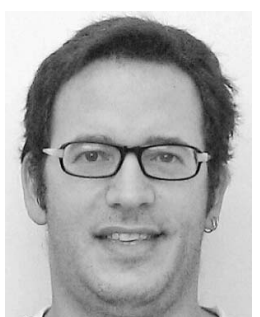

Gabriel Scalosub received the BSc degree in mathematics and philosophy from the Hebrew University of Jerusalem, Israel, in 1996, and the $\mathrm{MSc}$ and $\mathrm{PhD}$ degrees in computer science from the Technion - Israel Institute of Technology, Haifa, in 2002 and 2007, respectively. In 2008, he was a postdoctoral fellow at Tel-Aviv University, Israel, and in 2009, he was a postdoctoral fellow at the University of Toronto, Canada. In October 2009, he joined the Department of Communication Systems Engineering at Ben Gurion University of the Negev, Israel. He served on various technical program committees, including INFOCOM, IWQoS, IFIP-Networking, ICCCN, and WCNC. His research focuses on theoretical algorithmic issues arising in various networking environments, including buffer management, scheduling, and wireless networks. He is also interested in broader aspects of combinatorial optimization, online algorithms, approximation algorithms, and algorithmic game theory. 